\title{
Grado de Conocimiento de la Guía de Práctica Clínica de Trastornos Hipertensivos durante el Embarazo en una Empresa Social del Estado en Barranquilla (Atl. - Col) durante el 2014
}

\author{
Degree of Knowledge of the Clinical Practice of Hypertensive \\ Disorders during Pregnancy in a State Social Enterprise in \\ Barranquilla ( Atl - . Col) during 2014
}

\author{
Beleño Agudelo $\mathrm{C}^{1}$, Salazar Araujo $\mathrm{M}^{2}$, Mercado Rodríguez $\mathrm{L}^{3}$, Altahona Rodríguez $\mathrm{Z}^{4}$, Rodríguez López $\mathrm{J}^{5}$, Lastre Amell $\mathrm{G}^{5}$ \\ ${ }^{1}$ CooSalud EPS, ${ }^{2}$ Maestría en Auditoria y Sistemas de Calidad en Servicios de Salud - Universidad Simón Bolívar, ${ }^{3}$ Asociación Mutual Barrios Unidos de \\ Quibdo EPS, ${ }^{4}$ Maestría en Auditoria y Sistemas de Calidad en Servicios de Salud - Universidad Simón Bolívar, ${ }^{5}$ Universidad Simón Bolívar
}

\begin{abstract}
Resumen - En el presente estudio daremos una mirada práctica a la percepción de conocimiento sobre las guías de práctica clínica que tiene el personal de salud de la empresa social del estado hospital niño Jesús.

Objetivo: Evaluar el conocimiento de la guía de práctica clínica de trastornos hipertensivos en el embarazo, entre el personal de la salud en Empresa Social del Estado en el 2014.

Materiales y métodos: tipo de estudio descriptivo, exploratorio. Se utilizó un cuestionario diseñado para evaluar el conocimiento de los aspectos más relevantes en el manejo de las pacientes, con trastornos hipertensivos durante el embarazo contemplados en la Guía Práctica Clínica. Se realizó una validación de consistencia interna $\mathrm{kr}_{20}=74 \%$. Se evaluó al personal asistencial de la Empresa Social del Estado del servicio de ginecobstetricia: Ginecólogos, Médicos, Enfermeras y Auxiliares de enfermería. Los datos fueron tratados generalmente y se agruparon utilizando medidas de tendencia central y de agrupación.

Resultados: Se encontró que los profesionales con más alto grado académico, son los que porcentualmente conocían de la existencia de la GPC Emb _ HTA, en contraste a lo esperado, frente al personal encargado del cuidado del Paciente, sin embargo no existe una diferencia estadísticamente significativa entre el porcentaje de Respuestas Correctas del perfil profesional de Diagnóstico y Tratamiento con el perfil del Cuidado del Paciente.

Conclusión: se llegó al punto que a mayor nivel académico mayor conocimiento de las guías de práctica clínica y que como barreras se encuentran la falta de tiempo y falta de difusión de las guías.
\end{abstract}

Palabras Claves: Evaluación, Conocimiento, Guía de Práctica Clínica (GPC), Barreras, personal de salud, Trastornos Hipertensivos (Fuente: DeCS).

Correspondencia: Gloria Lastre Amell. Programa de Enfermeria, Facultad de Ciencias de la Salud. Universidad Simón Bolívar. Cr 59 \# 59 - 65. Barranquilla, Atlántico, Colombia. glastre@unisimonbolivar.edu.co.

Citar: Beleño Agudelo C, Salazar Araujo M, Mercado Rodríguez L, Altahona Rodríguez Z, Rodríguez López J, Lastre Amell G. Grado de Conocimiento de la Guía de Práctica Clínica de Trastornos Hipertensivos durante el Embarazo en una Empresa Social del Estado en Barranquilla (Atl. - Col) durante el 2014. Cienc e Innovación en Salud [Internet]. 2015 Jan 1;3(1):16-22. Available from: http://publicaciones.unisimonbolivar.edu.co:82/rdigital/ojs/index.php/innovacionsalud/article /view/233/230.

Parte de este trabajo se encuentran soportado en la investigación titulada «Grado de Conocimiento de la Guía de Práctica Clínica de Trastornos Hipertensivos durante el Embarazo en una Empresa Social del Estado en Barranquilla (Atl. - Col) durante el 2014»
Abstract - This study will give you a practical look at the perception of knowledge about clinical practice guidelines that health workers have social enterprise state hospital.

Objective: To assess the knowledge of the clinical practice guideline on hypertensive disorders in pregnancy among health personnel in the Social Enterprise 2014.

Methods: Study's type is descriptive and exploratory. A questionnaire designed to assess the knowledge of the most important aspects in the management of patients with hypertensive disorders during pregnancy contemplated in the Practical Clinical Guide were used. Validation of internal consistency $\mathrm{kr}_{20}=74 \%$ was performed. We assessed the caregiver of the State Social Enterprise gynecology service: Gynecologists, Physicians, Nurses and Nursing Assistants. Data was treated generally and pooled using measures of central tendency and grouping.

Results: We found that professionals with highest academic degree, are the percentage knew of the existence of the GPC Emb_HT, in contrast to expectations, compared to the staff caring for the patient, but there is not a statistically significant between the percentage of Correct Answers professional profile Diagnosis and Treatment with the profile of Patient Care.

Conclusion: Got to the point that the higher academic level greater knowledge of the clinical practice guidelines and as barriers are lack of time and lack of dissemination of the guidelines.

Keywords: Assessment, Knowledge, Clinical Practice Guideline (CPG), barriers, personal health, Hypertensive disorders. (Source: MeSH, NLM).

\section{INTRODUCCIÓN}

En la actualidad existe una creciente demanda para asegurar una mayor calidad al otorgar y recibir atención médica. Los servicios de salud enfrentan diferentes retos como por ejemplo variaciones frecuentes en la práctica clínica y aumento rápido de la generación de información de métodos diagnósticos y terapéuticos. En

tesis para obtener el título de Magíster Auditoria y Sistemas de Calidad en Servicios de Salud de la Universidad de Simón Bolivar (Colombia).

Recibido: Oct. 12 de 2014 / Modificado: Nov. 11 de 2014 / Aceptado: Nov. 21 de 2014 
este contexto surge una corriente de elaboración y uso de guías de práctica clínica basadas en evidencia científica, con el objeto de promover la excelencia en la atención médica (1).

Las guía de práctica clínica o guías clínicas son recomendaciones desarrolladas sistemáticamente para asistir a profesionales y pacientes en los cuidados de salud apropiados para determinadas condiciones clínicas (2). El desarrollo completo de buenas guías es un proceso que requiere tiempo, implica la elección de temas (de acuerdo con las prioridades), la planificación, el desarrollo en sí misma, la validación, diseminación, implementación, mantenimiento, evaluación de la adherencia y generalización de cada guía. Estas constituyen un conjunto de recomendaciones, basadas en la evidencia científica, que orientan la actividad médica frente a un paciente con determinada condición clínica. Deben basarse en la que se consideran las mejores evidencias científicas disponibles entre las diferentes opciones de diagnóstico y tratamiento (3).

La elaboración e implementación de las guías de práctica clínica tienen como fin, pasar del criterio basado en la opinión subjetiva sobre cuáles son los mejores procedimientos y métodos clínicos a priorizar aquellos que se fundamentan en datos experimentales procedentes de ensayos clínicos aleatorizados con diseño de alta calidad (4).

El desempeño de los profesionales de la salud está sujeto a variabilidad de una comunidad a otra, entre sitios de atención e incluso de un médico a otro. No todos los estilos de trabajo son correctos, pero se está en la obligación de encontrar cuales son los mejores. Para solucionar este problema, hay un interés creciente por la promoción, desarrollo y aplicación de las guías de práctica clínica, encaminadas a la prevención, diagnóstico y el tratamiento de diversas situaciones clínicas y susceptibles de vulnerabilidad no deseable y debe prestarse mayor atención a su implementación y efectividad en los diferentes escenarios de prácticas. No se toma con la misma seguridad una decisión clínica evaluada por una buena evidencia científica, que por una evidencia científica limitada o solo por la opinión de un experto aunque sea respetable pero que no proviene de los resultados de la investigación científica (5).

Los objetivos de desarrollo del milenio incluyeron el mejoramiento de la salud materna como una prioridad, sin embargo, su progreso no ha sido insuficiente para alcanzar las metas propuestas en los países en vías de desarrollo (6).

Producto de esta problemática la Organización Mundial de la Salud (OMS) ha invitado a los gobiernos a reafirmar su compromiso con la salud y los derechos reproductivos, y restablecerlos como prioritarios en materia sanitaria y de desarrollo. En esta dirección, hoy en día se considera que los estudios en salud reproductiva deben ser una prioridad (7).

Realizar seguimiento a los conocimientos y adherencia de protocolos y guía de práctica clínica en las instituciones prestadoras de servicio de salud para la atención materna perinatal, es una de las instrucciones dadas por el ministerio de la protección social considerando que garantizar la maternidad segura, con enfoque integral para el desarrollo pleno de la salud sexual y reproductiva, es una prioridad nacional, en donde se concentran esfuerzos en la reducción de las muertes maternas y perinatales por causas médicas y no médicas prevenibles e intervenirles, así mismo que la mortalidad materna es un indicador que expresa la situación de salud y calidad de vida de la población ya que advierte sobre las condiciones sociales, educativas, económicas familiares y personales de la mujeres y mismo, permite evaluar la calidad de los servicios de salud (6)

Según Duley (2009) más de medio millón de mujeres mueren cada año por problemas del embarazo, parto y puerperio y por complicaciones de estos eventos; y el 99\% de estos ocurren en países en vías de desarrollo, siendo estas complicaciones las principales causas de muerte en mujeres en edad reproductiva. En el caso particular de Colombia, la tasa de mortalidad materna para el 2007 fue de 76 muertes maternas por 100.000 nacidos vivos, ubicándola como la tercera causa de muerte en la mujer adulta joven (8).

Los trastornos hipertensivos del embarazo son causa importante de morbilidad grave, discapacidad crónica y muerte entre las madres, los fetos y los recién nacidos. En África y Asia, casi una décima parte de las defunciones maternas están relacionadas con estos trastornos; en América Latina, una cuarta parte de las muertes maternas se relacionan con esas complicaciones. Entre los trastornos hipertensivos que complican el embarazo, la pre-eclampsia y la eclampsia sobresalen como causas principales de morbilidad y mortalidad maternas y perinatales. La mayoría de las muertes causadas por la pre-eclampsia y la eclampsia se pueden evitar prestando asistencia oportuna y eficaz a las mujeres que acuden a consulta por estas complicaciones (9).

\section{MATERIALES Y MÉTODOS}

La población estuvo compuesta por los trabajadores del área asistencial la ESE Hospital Niño Jesús de 
Barranquilla. De esta se tomó una muestra a priori, restringida a los criterios de inclusión y exclusión. Como criterio de inclusión se pidió que fueran del Personal de la Salud que se encuentre asignado al pabellón de Ginecobstetricia, vinculado laboralmente con la institución por más de un año, profesionales que hayan aceptado participar en el estudio, no se tuvieron en cuenta el personal que se encontrara vinculado laboralmente por tercerización o Prestación de Servicio.

Se diseño del Instrumento, el cual fue validado a través del estadístico de kuder-richardson $\left(\mathrm{kr}_{20}\right)$ (10) el cual mide la consistencia interna de los instrumentos. Para la validación de la guía se utilizaron dieciocho profesionales de la salud y el valor obtenido fue del $\mathrm{kr}_{20}$ $=74 \%$.

\section{RESULTADOS}

La población estuvo compuesta por una muestra mixta de géneros. Donde hubo una predominancia del género femenino. El género femenino tuvo una representatividad del $84,85 \%$, siendo el género masculino una casi una sexta parte de esta representatividad, $15,5 \%$. Siendo la proporción sexual 6:1 para género femenino.

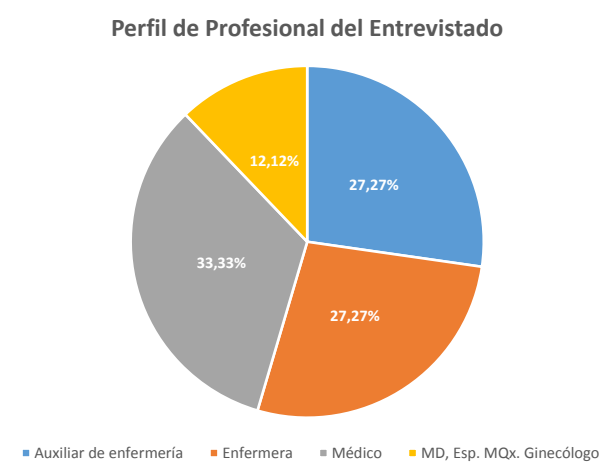

Ilustración 1 Gráfico circular de la distribución de los informantes por Perfil de Profesional del Entrevistado [Fuente: Datos del Estudio]

Dentro del pabellón de ginecobstetricia se encontraron dos tipos de profesionales del área de la salud, los dedicados al cuidado del paciente (Enfermeras y Auxiliares de Enfermería) y los dedicados al diagnóstico y tratamiento del paciente (Médicos y EspMQx en Ginecología).

Conocimiento, Actitudes en la toma de decisiones frente a la Existencia de la Guía de Práctica Clínica

Se interrogó a los informantes sobre si conocían la existencia de la Guía de Práctica Clínica de Trastornos Hipertensivos durante el Embarazo (GPCEmb_HTA), esto se realizó para todos los perfiles profesionales que se encontraban dentro del Pabellón de Ginecobstetricia de la ESE en mención.

Los resultados mostraron que el personal dedicado al cuidado del paciente (Auxiliares de Enfermería y Enfermeras) en un promedio de $44 \%$ si conocían la existencia de la existencia de la GPCEmb_HTA. Mientras que el personal de encargado del Diagnóstico $y$ Tratamiento, el 69,81\% conocía de la GPCEmb_HTA.

Tabla 1 Conocimiento de Existencia de Guías de acuerdo al Perfil del Profesional

\begin{tabular}{lcc}
\hline \hline Perfil de Profesional del Entrevistado & Frec. Obs. & Rep. \% \\
\hline Auxiliar de enfermería & 4 & $44,44 \%$ \\
Enfermera & 4 & $44,44 \%$ \\
Médico & 7 & $63,64 \%$ \\
MD, Esp. MQx. Ginecólogo & 3 & $75,00 \%$ \\
\hline \hline
\end{tabular}

[Fuente: Datos del Estudio]

Es de notar que los profesionales con más alto grado académico (MD, Esp. MQx), son los que porcentualmente conocían de la existencia de la GPCEmb_HTA (75\%). Un resultado que parece contradictorio, con lo encontrado la tendencia a nivel mundial que las GPC son de mayor conocimiento por parte del gremio dedicado al cuidado que de los médicos $(11,12,13)$.

Al indagar sobre los Criterios utilizados para la toma de decisiones se observa que los médicos sin Esp. MQx Ginecología basan sus decisiones en los conocimientos previos $(18,18 \%)$ pero mayoritariamente usan la Consulta a Colegas y/u Otros Profesionales (27,27\%), sin embargo muestran una utilización baja de las GPCEmb_HTA (18,18\%).

Tabla 2 Distribución de Frecuencia de acuerdo al Criterio de Toma de Decisiones

\begin{tabular}{llc}
\hline \hline $\begin{array}{l}\text { Criterio de Toma de } \\
\text { Decisiones }\end{array}$ & Perfil Profesional & $\begin{array}{l}\text { Rep. } \\
\text { Porcentual }\end{array}$ \\
\hline Conocimientos & Médico & $18,18 \%$ \\
& MD, Esp. MQx. & $0,00 \%$ \\
Ginecólogo & $0,00 \%$ \\
Experiencia & MD, Esp. MQx. & $25,00 \%$ \\
Consulta con colegas y & Ginecólogo & $27,27 \%$ \\
otros profesionales & MD, Esp. MQx. & $0,00 \%$ \\
\multirow{2}{*}{ Libros } & Ginecólogo & $18,18 \%$ \\
& Médico & $0,00 \%$ \\
Guía de práctica clínica & MD, Esp. MQx. & $18,18 \%$ \\
& Ginecólogo & $25,00 \%$ \\
\hline \hline [Fuente: Datos & Ginecólogo &
\end{tabular}

[Fuente: Datos del Estudio]

Al contrario, los MD, EspMQx Ginecología sus 
decisiones la basan en su experiencia de manejo clínico así como a la GPCEmb_HTA (25,00\%, respectivamente), dejando de lado totalmente a los conocimiento adquiridos en el pregrado y lo registrado en los libros así como la consulta a otro profesional $(0,00 \%$, respectivamente).

Teniendo en cuenta solo los que declararon conocer la GPCEmb_HTA, que indicó que el 54,55\% dijo conocer la existencia de una GPCEmb_HTA, se observa que todos (100\% informantes) dicen que adhiere por lo menos algunas veces a la GPCEmb_HTA, es decir que $46,45 \%$ se adhiere a una GPCEmb_HTA. Estos resultados son totalmente contradictorios, puesto el principal requisito para adherirse a una GPC es conocerla.

Tomando lo presentes resultados se excluyeron del análisis todas aquellas personas que no hayan referido conocer la GPCEmb_HTA, dado que sería imposible establecer si era cierta la respuesta. Para evitar el sesgo causado por ese fenómeno, se realizó lo planteado anteriormente.

De los que conocen la GPCEmb_HTA, las Auxiliares de Enfermería, Enfermeras y MD, Esp. MQx aplicaron Siempre y Casi Siempre la guía. En los Médicos hubo un porcentaje $(28,57 \%)$ que reconoció que solo Algunas Veces utiliza la GPCEmb_HTA.

Tabla 3 Frecuencia de Adherencia a la GPCEmb_HTA de los que si conocen la GPCEmb_HTA.

\begin{tabular}{lcccc}
\hline \hline $\begin{array}{l}\text { Perfil } \\
\text { Profesional }\end{array}$ & $\begin{array}{c}\text { Algunas } \\
\text { veces }\end{array}$ & $\begin{array}{c}\text { Casi } \\
\text { siempre }\end{array}$ & Siempre & TOTAL \\
\hline $\begin{array}{l}\text { Auxiliar de } \\
\text { enfermería }\end{array}$ & $0(0,00 \%)$ & $3(75,00 \%)$ & $1(25,00 \%)$ & $4(100 \%)$ \\
$\begin{array}{l}\text { Enfermera } \\
\text { Médico }\end{array}$ & $0(0,00 \%)$ & $1(25,00 \%)$ & $3(75,00 \%)$ & $4(100 \%)$ \\
$\begin{array}{l}\text { MD, Esp. } \\
\begin{array}{l}\text { MQx. } \\
\text { Ginecólogo }\end{array}\end{array}$ & $0(0,00 \%)$ & $2(66,67 \%)$ & $1(33,33 \%)$ & $3(100 \%)$ \\
\hline \hline
\end{tabular}

[Fuente: Datos del Estudio]

\section{Conocimiento y Práctica Empírica de la} GPCEMb_HTA

A los informantes se les realizó un test de conocimiento basado en la GPCEmb_HTA, con la finalidad de conocer que tanto ponían empíricamente en práctica los conocimientos referidos, se les calificó las respuestas y/o procedimientos a seguir. Se cuantificaron solo las respuestas correctas. De acuerdo a la GPCEmb_HTA para cada Perfil Profesional los requerimientos pueden variar. Se estandarizaron los datos a Porcentaje de Respuestas correctas para poder realizar las comparaciones pertinentes.

En promedio todos los informantes respondieron el $70,59 \pm 15,42 \%$ de las respuestas correctamente, presentándose un mínimo de 45,45\% y un máximo de $100 \%$ de las respuestas correctas. Solo los MD, Esp. MQx obtuvieron un $100 \%$ de las respuestas, lo cual indica el nivel de preparación de estos profesionales, sin embargo, hubo un solo profesional de este perfil que obtuvo un muy bajo porcentaje de respuestas correctas, solo un $48,48 \%$.

Nivel de Conocimiento de los Procedimientos descritos en la Guía de Practica Clínica

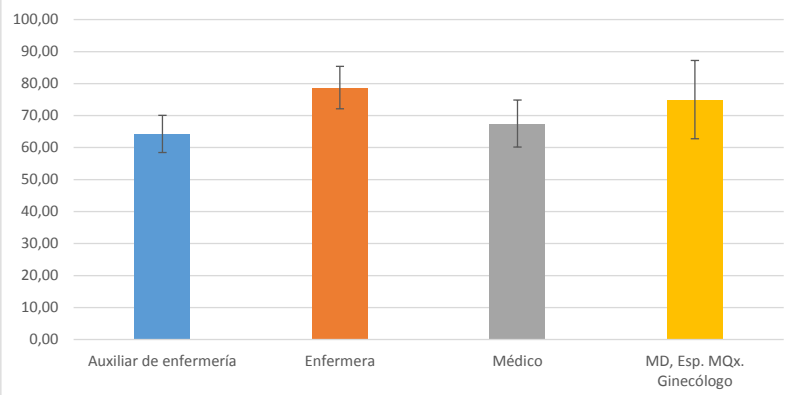

llustración 2 Gráfico de Barras Comparativo entre el Promedio Porcentual \pm Desviación Estándar de las Respuestas Correctas por Perfil Profesional [Fuente: Datos del Estudio].

Comparativamente, las Enfermeras el mejor promedio de respuesta correctas así como la desviación estándar más baja $(78,74 \pm 13,30 \%)$, seguido de los MD, Esp. MQx con $75 \pm 24,42 \%$. Las Auxiliares de enfermería fueron las que obtuvieron el porcentaje de asertividad más bajo $(64,25 \pm 11,66 \%)$, sin embargo los Médicos se ubicaron a apenas a 3,24\% de los Tecnólogos Profesionales en esta área de atención con el $67,49 \pm 14,73 \%$ de respuestas correctas.

De particular interés aquí son el sesgo estandarizado y la curtosis estandarizada, las cuales pueden utilizarse para determinar si la muestra proviene de una distribución normal y de este modo definir la prueba estadística a utilizar, para este caso particular un Análisis de Varianza (ANOVA). El valor del sesgo estandarizado y de curtosis estandarizada se encuentra dentro del rango esperado (-2 y 2) para datos provenientes de una distribución normal, por lo tanto la prueba de elección es la ANOVA.

Tabla 4 ANOVA para Porcentaje de Respuestas Correctas por Perfil del Entrevistado

\begin{tabular}{lccccc}
\hline \hline Fuente & $\begin{array}{c}\text { Sumade } \\
\text { Cuadrados }\end{array}$ & gl & $\begin{array}{c}\text { Cuadrado } \\
\text { Medio }\end{array}$ & $\begin{array}{c}\text { Razón- } \\
\boldsymbol{F}\end{array}$ & $\begin{array}{c}\text { Valor- } \\
\boldsymbol{P}\end{array}$ \\
\hline Entre grupos & 1143,03 & 3 & 381,009 & 1,71 & 0,1868 \\
Intra grupos & 6461,52 & 29 & 222,811 & & \\
Total (Corr.) & 7604,54 & 32 & & & \\
\hline \hline
\end{tabular}

La tabla ANOVA descompone la varianza de los datos en dos componentes: un componente entre-grupos y un 
componente dentro-de-grupos. La razón-F, que en este caso es igual a 1,71001, es el cociente entre el estimado entre-grupos y el estimado dentro-de-grupos. Puesto que el valor-P de la razón-F es mayor o igual que 0,05 , no existe una diferencia estadísticamente significativa entre las medias de las 4 variables con un nivel del 95,0\% de confianza.

Se aplicó un procedimiento de comparación múltiple para determinar cuáles medias son significativamente diferentes de otras. El método empleado actualmente para discriminar entre las medias es el procedimiento de diferencia mínima significativa (LSD) de Fisher. Encontrándose una diferencia estadísticamente significativa entre el porcentaje de Respuesta Correctas entre el perfil profesional Auxiliar de Enfermería y Enfermera esta diferencia está dada a un 95,0\% de confianza.

Tabla 5 Pruebas de Múltiple Rangos Método: LSD al 95,0\% de Confianza

\begin{tabular}{llll}
\hline \hline Contraste & Sig. & Diferencia & +/- Límites \\
\hline Auxiliar de enfermería-Enfermera & $*$ & $-14,4911$ & 14,392 \\
Auxiliar de enfermería - & & $-10,7467$ & 18,346 \\
Ginecólogo & & $-3,24121$ & 13,722 \\
Auxiliar de enfermería-Médico & & 3,74444 & 18,346 \\
Enfermera - Ginecólogo & & 11,2499 & 13,722 \\
Enfermera - Médico & & 7,50545 & 17,825 \\
Ginecólogo - Médico
\end{tabular}

* indica una diferencia significativa. [Fuente: Datos del Estudio]

Al contrastar nuestros resultados con lo de otros investigadores $(14,15)$ las enfermeras tienen un mayor uso de la GPC que los médicos, por lo tanto una mayor conocimiento empírico de la GPC. Esto se evidencia en el análisis de frecuencia, sin embargo el ANOVA demostró que esta diferencia es nula.

\section{Barreras de Acceso y Adherencia a la GPCEmb_HTA}

Así mismo, se indagó sobre las barreras que propendían por un no uso de la GPCEmb_HTA, dado que no existió una diferencia en el conocimiento y manejo empírico de la GPCEmb_HTA, los resultados se globalizaron con la finalidad de presentar una información consolidada.

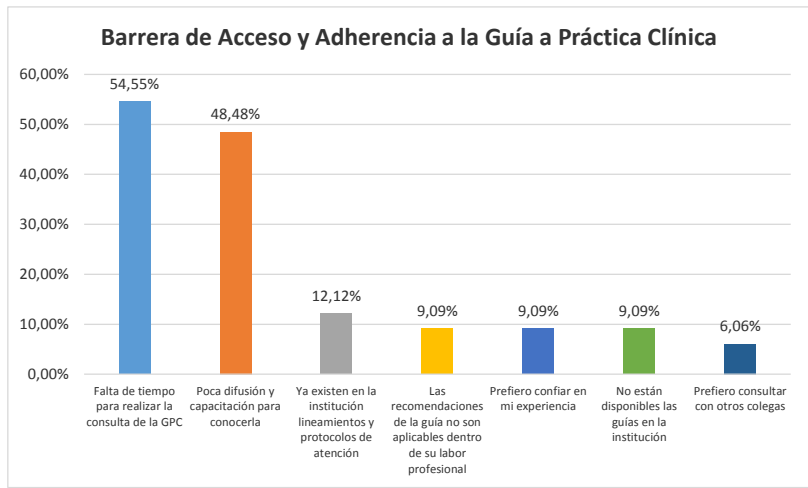

Ilustración 3 Diagrama de Barras Comparativo de las principales barreras de acceso y adherencia a la GPCEmb_HTA. [Fuente: Datos del Estudio]

Dentro de las opciones, las dos de mayor referencia fueron Falta de tiempo para realizar la consulta de la GPCEmb_HTA y Poca difusión y capacitación para conocerla, con 54,55 y $48,48 \%$, respectivamente.

Tabla 6 Frecuencia de las principales barreras de uso y acceso a la GPCEmb_HTA.

\begin{tabular}{|c|c|c|}
\hline $\begin{array}{l}\text { Principal Barrera de Adherencia a la Guía de } \\
\text { Práctica Clínica }\end{array}$ & $\begin{array}{l}\text { Frec. } \\
\text { Obs. }\end{array}$ & $\operatorname{Rep} \%$ \\
\hline $\begin{array}{l}\text { Falta de tiempo para realizar la consulta de la } \\
\text { GPCEmb_HTA }\end{array}$ & 18 & $54,55 \%$ \\
\hline Poca difusión y capacitación para conocerla & 16 & $48,48 \%$ \\
\hline $\begin{array}{l}\text { Ya existen en la institución lineamientos y } \\
\text { protocolos de atención }\end{array}$ & 4 & $12,12 \%$ \\
\hline $\begin{array}{l}\text { Las recomendaciones de la guía no son } \\
\text { aplicables dentro de su labor profesional }\end{array}$ & 3 & $9,09 \%$ \\
\hline Prefiero confiar en mi experiencia & 3 & $9,09 \%$ \\
\hline No están disponibles las guías en la institución & 3 & $9,09 \%$ \\
\hline Prefiero consultar con otros colegas & 2 & $6,06 \%$ \\
\hline
\end{tabular}

Estos resultados indican con claridad la misma apreciación de otros estudios similares, que es, a los profesionales de la salud se le debe facilitar el tiempo de capacitación (16). Al indagar sobre la posible relación entre las Barreras de Accesos y Adherencia a la GPCEmb_HTA con respecto al Nivel de Conocimiento y Práctica Empírica de la GPCEmb_HTA, lo cual se realizó a través de una Prueba de $\chi^{2}$. Esta tabla muestra los resultados de la prueba de hipótesis ejecutada para determinar si se rechaza, o no, la idea de que las clasificaciones del Nivel de Conocimiento y Práctica Empírica de la GPCEmb_HTA y Barreras de Accesos y Adherencia a la GPCEmb_HTA son independientes.

Puesto que el valor-P es mayor o igual que 0,05 , no se puede rechazar la hipótesis de que Nivel de Conocimiento y Práctica Empírica de la GPCEmb_HTA y Barreras de Accesos y Adherencia a la GPCEmb_HTA son 
independientes con un nivel de confianza del 95\%. Por lo tanto, el valor observado de Nivel de Conocimiento y Práctica Empírica de la GPCEmb_HTA para este caso en particular, pudiera no tener relación con su valor en Barreras de Accesos y Adherencia a la GPCEmb_HTA.

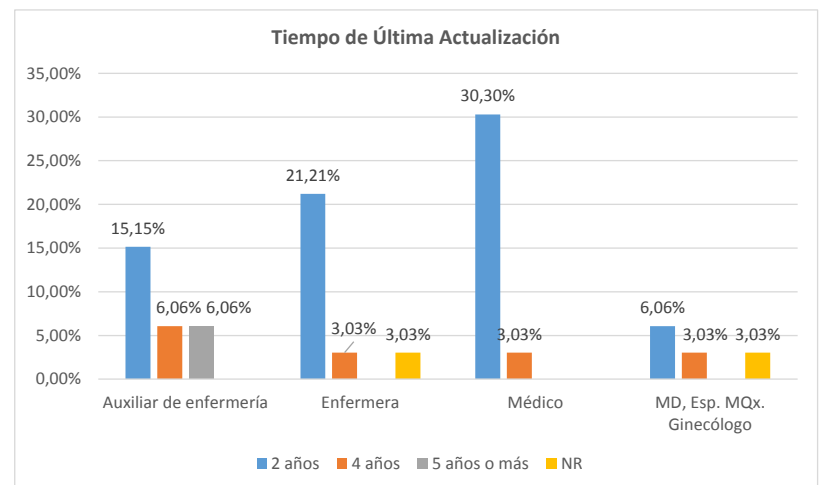

Ilustración 4 Gráfico de Barras sobre la última actualización por perfil profesional [Fuente: Datos del Estudio]

Por último, los informantes mencionaron en un $60,61 \%$ que para lograr la utilización de GPCEmb_HTA se hace necesario que haya un programa de capacitación de la GPCEmb_HTA. Otra posibilidad mencionada en un $21,21 \%$ es que los directivos de la institución promuevan el conocimiento y uso de la GPCEmb_HTA.

\section{DISCUSIÓN}

En cuanto a la proporción sexual el resultado fue el esperado dado lo expresado por Gómez (2002) (17) en el cual expresa que el aumento de la vinculación laboral de la mujeres en el área de salud es mayor en los últimos 30 años que la de los hombres. Esta relación laboral está dada en el área de la salud por las necesidades del servicio en salud, especialmente en el área de la ginecobstetricia, el cual necesita que el profesional dedique mayor cuidado y logre identificarse con el paciente, es decir que el profesional se adaptable; esta adaptación es más fácil para el género femenino de acuerdo a lo mencionado por Graf \& Diogo en el 2009 (18).

La proporción de los encargados del Cuidado del Paciente fue $54,5 \%$ mientras que de los encargados del Diagnóstico y Tratamiento fue del 45,5\%. Esta distribución de profesionales es realista y ajusta a las necesidades del servicio para este pabellón (19). Así mismo la distribución del personal dedicado al cuidado del paciente es el apropiado (20), esta aseveración se da puesto que en el sitio de estudio la planeación del personal se realiza de acuerdo a los criterios de ley.

La relación planteada en los resultados, también se ha visto evidenciada en diferentes ocasiones por Corona \&
Fonseca $(21,22,23)$ y referenciado previamente en 1998 por Robbins (24). Y se explica desde la creación del currículo académico en los programas de post-grado, en el cual al residente se le instruye a desarrollar un criterio de manejo apegado a una GPC nutrida con su experiencia en terreno propiamente dicho.

Es probable que la creencia que los perfiles profesionales encargados del cuidado y manejo del paciente tenía un mejor conocimiento que los perfiles profesionales responsables del diagnóstico y tratamiento y está estaba fundamentada en el número de horas contacto del profesional-paciente (25). Otra posibilidad es que las GPC fueron diseñadas para el personal de Manejo y Cuidado del Paciente. Sin embargo en nuestros resultados todos los profesionales tenían un manejo y conocimiento empírico de la GPCEmb_HTA.

La mayoría de los profesionales relacionados en el estudio tenían una actualización de servicios en salud para el área de ginecobstetricia menor a dos años. Lo que en teoría debiera ser considerado al momento de evaluar el tipo de respuesta, dado que son profesionales en servicio activo y actualizado. Esta situación ya ha sido evidenciada por Patiño et al. (2012) $(26,27,28)$.

\section{REFERENCIAS BIBLIOGRÁFICAS}

1. Constantino-Casas $P 1$, Viniegra-Osorio A, MedécigoMicete C, del Pilar Torres-Arreola L, ValenzuelaFlores A. «El potencial de la guías de práctica clínica para mejorar la calidad de la atención». RevMedInstMex Seguro Soc. 2009 Ene - Feb; 47(1):103-8.

2. Grupo de trabajo de la Guía de Práctica Clínica sobre Atención al Parto Normal. Guía de Práctica Clínica sobre la Atención al Parto Normal. Plan de Calidad para el Sistema Nacional de Salud del Ministerio de Sanidad y Política Social. Agencia de Evaluación de Tecnologías Sanitarias del País Vasco (OSTEBA). Agencia de Evaluación de Tecnologías Sanitarias de Galicia (Avalia-t). 2010. Guías de Práctica Clínica en el SNS: OSTEBA № 2009/01

3. Espinosa Brito Alfredo, Del Sol Padrón Luis, Espinosa Brito Alfredo, Garriga Valdés José, Viera Valdés Brandy. "Guías de práctica clínica. Ventajas y desventajas: Una propuesta de indicadores». MediSur. 2009 Oct; 7(5): 44-47.

4. Moreno Rajadel RE, Carlos Álvarez Li F. «Adherencia a la guía de práctica clínica en la cirugía funcional del septo nasal». RevCalidAsist. 2008 May; 23 (3): 12630.

5. Toledo Fernández Ana Margarita, Cabrera Cruz Niviola, Arteaga García Amaylid, Mejías Sánchez 
Yoerquis. "Calidad de las guías de práctica clínica cubanas». Rev Cubana Salud Pública. 2011 Sep; 37(3): 349-358

6. OMS. «Informe Objetivos de desarrollo del milenio» Organización Mundial de la Salud, 2008. pp 1-56.

7. Organización Mundial de la Salud. «Subsanar la desigualdad en una generación: Informe final de la Comisión sobre Determinantes Sociales de la Salud». Organización Mundial de la Salud. 2009. p. 1-40.

8. Laza Vásquez C., Sánchez Vanegas G. Indagación desde los conocimientos, actitudes y prácticas en salud reproductiva femenina: algunos aportes desde la investigación. Enferm. glob. 2012 Abr ; 11(26): 408-415.

9. Giorgi, M. A., Borracci, R. A., Calderón, G., Manente, D., Mulassi, A., Piñeiro, D. J., \& Dones, W. SurveyonClinicalPracticeGuidelines Use among Latin American Cardiologists. Argentine Journal of Cardiology, 2014, 80(2), 108-113.

10. Morales-Vallejo P, «Estadísticas aplicada a las Ciencias Sociales - La fiabilidad de los test y escalas», Universidad Pontificia Comillas, España, 2007.

11. GoncalvesICM, GoncalvesMJ. Conocimientos, actitudes y prácticas de las enfermeras y los medicos acerca de la transmisión vertical de la hepatitis $B$. Rev. Latino-Am. Enfermagem. 2013 Oct; 21(5): 10301038.

12. Bueno N. F, Sáez C. J, Ocaranza B. M, Conejeros R. C, Vaccarezza P. I, Borquez V. Pamela et al. Tratamiento Médico del Embarazo Ectopico. Rev. chil. obstet. ginecol. 2002; 67(3): 173-179.

13. Prieto-Miranda Sergio E., Calixto-Bello RA., JiménezBernardino CA., Guerrero-Quintero LG. Evaluación de conocimientos sobre el manejo de ventilación mecánica en médicos residentes. FEM. 2013 Dic; 16(4): 203-213.

14. Reyes López $M$, Pérez Gómez JM, García Olmos $P$, Borrell Carrió F, Gracia Guillén D., Relaciones entre profesionales sanitarios., Medicina Clínica, 117(9), 2001; 339-350.

15. Álvarez $\mathrm{CA}$, Cortés JA, Gómez $\mathrm{CH}$, Fernández JA, SossaMP, Beltrán F, Izquierdo GM, Andrade FM, Ortiz G, Padilla A., Guías de práctica clínica para la prevención de infecciones intrahospitalarias asociadas al uso de dispositivos médicos., Revista Infectio, 2010, 14(4); 292-308.

16. Nogales-Gaete Jorge. Introducción de innovaciones en la práctica clínica y capacitación médica: Comentario en relación a la reciente incorporación de la enfermedad vascular cerebral isquémica aguda, en el Régimen de Garantías Explícitas en Salud - Auge. Rev. chil. neuropsiquiatr. 2006 Dic; 44(4): 242-248.

17. Gómez Gómez Elsa. Equidad, género y salud: retos para la acción. Rev Panam Salud Publica. 2002 June; 11(5-6): 454-
461.

18. Graf, L \&Diogo, M. Projeçõesjuvenis: visõesocupacionais e marcas de gênero. Rev. bras. orientac. prof. 2009, 10(1): 71-82.

19. Restrepo, N., N. Velasco, and C. A. Amaya. Planeación de personal para las diversas áreas que componen un centro médico: Un caso de aplicación. Los cuadernos de PYLO Logística Hospitalaria. 2009.

20. Giraldo C. Las necesidades del cuidado de enfermería: criterios para definir requerimientos de personal de enfermería. Investigación y Educación en Enfermería, 2000, 18(1); 49 - 68.

21. Corona Martínez LA, Fonseca Hernández M. La toma de decisiones médicas y su formación como habilidad profesional. EducMedSuper. 2003 Dic; 17(4)

22. Corona Martínez LA, Fonseca Hernández M. La toma de decisiones médicas como la habilidad profesional esencial en la carrera de Medicina: una preocupación de todos. MediSur. 2010 Feb; 8(1): 42-45.

23. Corona Martinez LA, Fonseca Hernández M. Contribución de la teoría sobre la toma de decisiones a la atención médica integral: Lo que hemos aprendido de la atención a estos enfermos en Cienfuegos. MediSur. 2009 Oct; 7(5): 14-16.

24. Robbins, S. P. Toma individual de decisiones. En: Fundamentos de comportamiento organizacional: Toma individual de decisiones. 1998.

25. Plaza V, Bolívar I, Jordi Giner, María Antonia Llauger, Antolín López-Viña, José Antonio Quintano, Joaquín Sanchis, Montserrat Torrejón, José Ramón Villa, Opinión, conocimientos y grado de seguimiento referidos por los profesionales sanitarios españoles de la Guía Española para el Manejo del Asma (GEMA). Proyecto GEMA-TEST, Archivos de Bronconeumología, 2008 May, 44(5): 245-251.

26. Patiño Perez A, Torres M, Pinzón Junca A., Evaluación de la Efectividad de un Plan de Mejoramiento de la Implementación de la Guía de Práctica Clínica de Anticoagulación Terapéutica Oral Intrahospitalaria en el Hospital Universitario de la Samaritana Basada en Indicadores de Desempeño de la Guía (Estudio Antehus). Tesis de Especialidad en Medicina Interna., Universidad Javeriana, 2012.

27. Barnet-Dinamarca, R. Capacitación en servicio"-Guía del facilitador para el personal de salud del primer nivel de atención. In Capacitación en servicio: guía del facilitador para el personal de salud del primer nivel de atención. UPCH. 1997.

28. Gutiérrez M, Delfín E., Guías de práctica clínica: su validez en la práctica médica en una institución prestadora de salud de primer nivel de atención en el 2007. Tesis Doctoral., Universidad CES., 2013.

Las opiniones que contenga este artículo son de su(s) autor(es) y no necesariamente representan la opinión oficial de la Universidad Simón Bolívar, la Revista Ciencia e Innovación en Salud o de sus autoridades. 